\title{
CRYPTANALYSIS OF A MODIFIED ROTOR MACHINE
}

\author{
Peer Wichmann \\ Universität Karlsruhe \\ Institut für Algorithmen u. kognitive Systeme
}

\section{Introduction}

In some operating systems one can find a modified rotor machine as a file crypt. The difference to a normal rotor machine can be characterized in two points:

- First difference : A different rotor model.

In general we have:

$$
\text { output }:=\text { permutation(input+position)-position }
$$

here we have:

$$
\text { output }:=\text { permutation(input }+ \text { position) }
$$

We guess the main purpose of this modification is to speed up the encryption.

- Second difference: An input based step function

$$
\text { (new position) := (old position) }+ \text { (output of previous rotor) }
$$

Because of this two modifications the input to the permutation and the new position are equal and do not have be computed separately.

In our description we use the following name conventions:

$a[i]$ plaintext at time $i(i=1,2,3, \ldots)$,

$c[i]$ ciphertext at time $i(i=1,2,3, \ldots)$,

$n$ is the number of rotors, 
$P_{k}$ stands for the permutation on the $k$-th rotor,

$A:=\{0,1, \ldots, M-1\}$ is the enciphered alphabet (normally one would think of $M=256$ for byte encryption),

$a_{j}[i]$ and $b_{j}[i]$ are intermediate sequences used to define the position and the output of each rotor. $(j=0,1,2, \ldots, n)$

Using this conventions we can describe the encryption as follows:

- Interface to the recursion scheme:

$$
\begin{aligned}
b_{j}[0] & :=0 \quad j=1,2, \ldots, n \\
a_{0}[i] & :=a[i] \quad i=1,2,3, \ldots \\
c[i] & :=a_{n}[i] \quad i=1,2,3, \ldots
\end{aligned}
$$

- Recursion scheme:

$$
\begin{aligned}
& b_{j}[i]:=a_{j-1}[i]+b_{j}[i-1] \bmod M j=1,2, \ldots, n i=1,2,3, \ldots \\
& a_{j}[i]:=\quad P_{j}\left(b_{j}[i]\right) \quad j=1,2, \ldots, n \quad i=1,2,3, \ldots
\end{aligned}
$$

Perhaps, the following picture is more convenient:

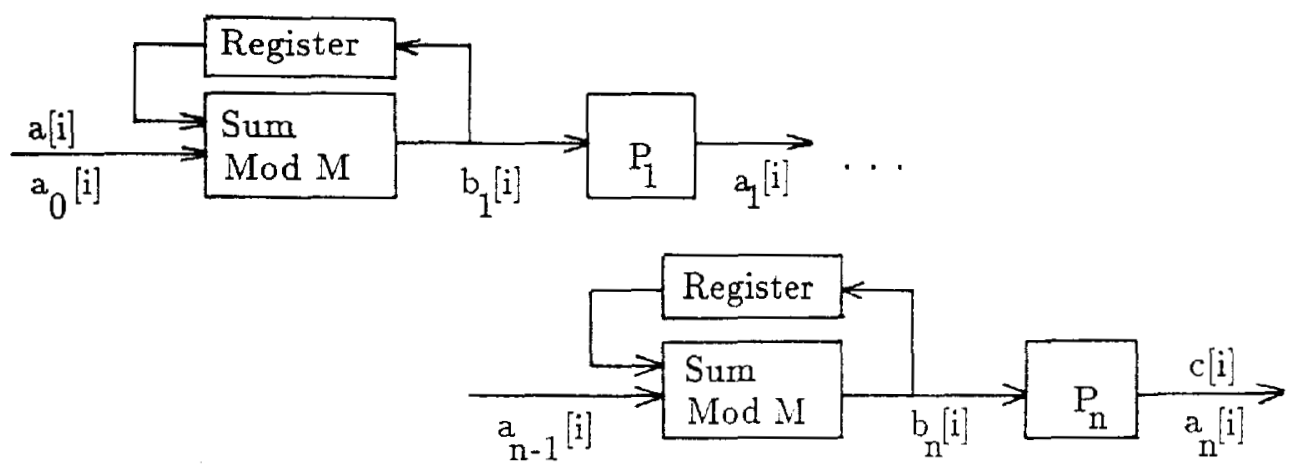

The key is formed by the $n$ permutations $P_{1}, \ldots, P_{n}$. The number of keys is limited by isomorphisms:

Let $h: A \rightarrow A$ be a group isomorphism on $(A,+)$ and $h^{-1}$ the inverse isomorphism.

If $P_{i}$ is substituted by $h\left(P_{i}\right)$ and $P_{i+1}$ is substituted by $P_{i+1}\left(h^{-1}\right)$ ( $i \in\{1,2, \ldots, n-1\}$ ), the same encryption is realized (because $\left.h^{-1}(h(x)+h(y))=h^{-1}(h(x+y))=x+y\right)$. 
$h$ is bijective on $A$ and thus $h$ is a permutation on $A$. Because of this $h\left(P_{i}\right)$ and $P_{i+1}\left(h^{-1}\right)$ are permutations too.

There are $\phi(M)$ different isomorphisms and $n-1$ possible positions where an isomorphism can be inserted. Thus the key space is reduced by the factor $\phi(M)^{n-1}$.

The remaining keys are truly different. This is proved by the fact that the key can be deduced within a chosen ciphertext attack (discussed below) with the exception of the isomorphisms mentioned above. The number of usable keys is again reduced because the machine is self synchronizing when operating in deciphering mode. It is useful to consider permutations as not significantly different if they can be reached by cyclic shifts. Thus the number of keys is reduced by the factor $M_{n}$.

The total number is: $\frac{M^{n}}{\left(M^{n} * \phi(M)^{n-1}\right)}$ (where $\phi$ is the Euler phi-function)

To characterize the deciphering mechanism we define a set of recursive functions as follows:

$$
\begin{aligned}
f_{n}(i) & :=c[i] \\
& \vdots \\
f_{r}(i) & :=P_{r+1}^{-1}\left(f_{r+1}(i)\right)-P_{r+1}^{-1}\left(f_{r+1}(i-1)\right) \\
& \vdots \\
f_{1}(i) & :=P_{2}^{-1}\left(f_{2}(i)\right)-P_{2}^{-1}\left(f_{2}(i-1)\right) \\
f_{0}(i) & :=P_{1}^{-1}\left(f_{1}(i)\right)-P_{1}^{-1}\left(f_{1}(i-1)\right)
\end{aligned}
$$

$f_{r}(i)$ deciphers the last $n-r$ rotors and calculates the value $a_{r}[i]$. There is one thing that should be mentioned:

$f_{\tau}(i)$ depends on the last $n+1-r$ ciphertext bytes: $c[i], c[i-1], \ldots, c[i-$ $n+r]$.

The flowchart for the deciphering mechanism looks somewhat different because the feedback loops are substituted by delay elements: 

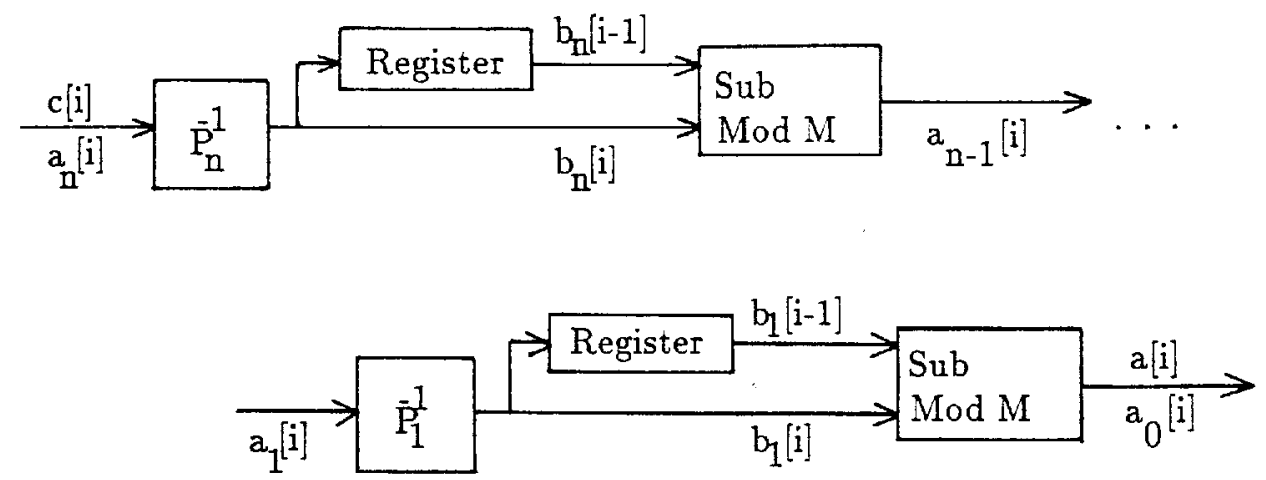

It is easy to see, that the machine is self-synchronizing when operating in deciphering mode. In the deciphering scheme one can also see what seems to be the main problem of the cipher:

\section{Constant ciphertext effect}

If the machine is fed with a constant ciphertext we have after $n$ characters $b_{j}[i]=P_{j}^{-1}(0)(j=1,2, \ldots, n-1)$. Thus the machine can be forced into a well defined state only by a given ciphertext and without knowing the key (the permutations). Later we will show that this fact makes it possible to easily break the cipher with a chosen-ciphertext-attack.

Proof of constant ciphertext effect:

Because of the constant ciphertext for each $j=2, \ldots, n$ the input to $f_{j}(i)$ is equal to the input of $f_{j}(i-1)$. So we have:

$$
\begin{array}{ll}
f_{j}(i)=f_{j}(i-1) & \Leftrightarrow \\
P_{j}^{-1}\left(f_{j}(i)\right)=P_{j}^{-1}\left(f_{j}(i-1)\right) & \Leftrightarrow \\
P_{j}^{-1}\left(f_{j}(i)\right)-P_{j}^{-1}\left(f_{j}(i-1)\right)=0=f_{j-1}(i)=a_{j-1}[i]=P_{j}^{-1}\left(b_{j-1}[i]\right) & \Rightarrow \\
f_{j}(i)=0 \quad j=1,2, \ldots, n-1 & \\
b_{j}[i]=P_{j}^{-1}(0) \quad j=1,2, \ldots, n-1 . &
\end{array}
$$




\section{Attacks}

\subsection{Ciphertext-only-attack}

We suppose it is very difficult to break the cipher under this conditions. The easiest way we found is to make a statistical analysis over $M^{n+1}$ different elements. Generally $M^{n+1}$ is very large and thus this attack will usually be impossible.

\subsection{Given-plaintext-attack}

A solution using backtracking may be possible but we found that even for small $M$ 's and $n$ 's the searching tree is much too deep and too wide. Another possibility is a solution using equations. With a given plaintext all $b_{1}[i]$ are given too. We have:

$$
b_{1}[i]=P_{1}^{-1}\left(f_{1}(i)\right)
$$

With $b_{1}[i]=b_{1}[j]$ we get:

$$
\begin{gathered}
P_{1}^{-1}\left(f_{1}(i)\right)=P_{1}^{-1}\left(f_{1}(j)\right) \Leftrightarrow\left(P_{1}^{-1} \text { is bijective }\right) \\
f_{1}(i)=f_{1}(j)
\end{gathered}
$$

with a little luck it is possible to find a position $j$ with $c[j]=c[j+1]=\ldots=$ $c[j+n-1]$ ( $n$ constant ciphertext chars). Because of the constant ciphertext effect we have $f_{1}(j)=0$ and get:

$$
\begin{aligned}
P_{2}^{-1}\left(f_{2}(i)\right)-P_{2}^{-1}\left(f_{2}(i-1)\right) & =0 & & \Leftrightarrow \\
P_{2}^{-1}\left(f_{2}(i)\right) & =P_{2}^{-1}\left(f_{2}(i-1)\right) & & \Leftrightarrow\left(P_{2}^{-1} \text { is bijective }\right) \\
f_{2}(i) & =f_{2}(i-1) & & \Leftrightarrow \\
P_{3}^{-1}\left(f_{3}(i)\right)-P_{3}^{-1}\left(f_{3}(i-1)\right) & =P_{3}^{-1}\left(f_{3}(i-1)\right)-P_{3}^{-1}\left(f_{3}(i-2)\right) & &
\end{aligned}
$$

It can be easily shown that the above trick does not work a second time. We found no further way to simplify the equations. Thus (treating $P_{3}^{-1}\left(f_{3}(i)\right)$ as an unknown) a linear system with $M^{n-2}$ unknowns has to be solved. It will usually be impossible to do this within an appropriate amount of time. This attack does not use the the internal structure of these 'unkowns'. Thus it may be possible to solve the equations much faster and much easier. 


\subsection{Chosen-plaintext-attack}

The idea is to feed the machine with a constant (zero) plaintext and to wait until the output becomes cyclic. To analyze the output we first consider the output of the first $n-1$ rotors $\left(a_{n-1}[i]\right)$. Let $m$ be the length of a cycle of $a_{n-1}[i]$. Let $s$ be the sum over a cycle of $a_{n-1}[i]: s:=a_{n-1}[1]+\ldots+a_{n-1}[m]$. Suppose $s$ and $M$ are coprime. Then the length of a complete output cycle is $s * M$. We have $c[i+j * m]=P_{n}\left(b_{n-1}[i]+j * s\right)$. Because $s$ and $M$ are coprime $j * s$ covers whole $A$. Because of this $c[i+j * m]=P_{n}\left(b_{n-1}[i]+j * s\right)$ covers $A$ too. Now $c[i+j * m]$ run through the same cycle for each $i$ (except for the starting point). If we use s as the '1-element' in $A$ we get:

$$
\begin{aligned}
P_{n}(0) & =c[1] \\
P_{n}(1) & =c[1+m] \\
& \vdots \\
P_{n}(M-1) & =c[1+m *(M-1)]
\end{aligned}
$$

Now the last rotor is completely known (except for rotation identical rotors).

This is only if $s$ and $M$ are coprime. If they are not coprime it is usually impossible to split the output into cycles covering $A$. Seeing a complete output cycle we can tell if it is possible to extract the last rotor. If it is impossible we have to restart the attack with a short random sequence before the zero plaintext to put the machine in an undefined state. The restart of the attack does not require the machine to be reset, because we simply want to put the machine into an undefined state. If the last rotor is known we can eliminate him from the equations and restart the whole attack to get the next rotor. After all rotors are known we need to compute the initial position $b_{i}[0]$ of each Iotor. To do this we encipher a random sequence $a[1], \ldots, a[n]$ and get the ciphertext $c[1], \ldots, c[n]$. Using the defining equations we now can compute the values in the following order: 


$$
\begin{array}{ll}
a_{n}[1], a_{n}[2], \ldots, a_{n}[n], & \left(a_{n}[i]=c[i]\right) \\
b_{n}[1], b_{n}[2], \ldots, b_{n}[n], & \left(b_{n}[i]=P_{n}^{-1}\left(a_{n}[i]\right)\right) \\
a_{n-1}[2], a_{n-1}[3], \ldots, a_{n-1}[n], & \left(a_{n-1}[i]=b_{n}[i-1]-b_{n}[i]\right) \\
\vdots & \\
b_{k}[n+1-k], b_{k}[n+2-k], \ldots, b_{k}[n], & \left(b_{k}[i]=P_{k}^{-1}\left(a_{k}[i]\right)\right) \\
a_{k-1}[n+2-k], a_{k-1}[n+3-k], \ldots, a_{k-1}[n], & \left(a_{k-1}[i]=b_{k}[i-1]-b_{k}[i]\right) \\
\vdots & \\
b_{1}[n], & \left(b_{1}[i]=P_{1}^{-1}\left(a_{1}[i]\right)\right) \\
b_{1}[n-1], b_{1}[n-2], \ldots, b_{1}[0], & \left(b_{1}[i]=b_{1}[i+1]-a[i+1]\right) \\
a_{1}[n-1], a_{1}[n-2], \ldots, a_{1}[0], & \left(a_{1}[i]=P_{1}\left(b_{1}[i]\right)\right) \\
\vdots & \\
b_{k}[n-k], b_{k}[n-k-1], \ldots, b_{k}[0], & \left(b_{k}[i]=b_{k}[i+1]-a_{k-1}[i+1]\right) \\
a_{k}[n-k], a_{k}[n-k-1], \ldots, a_{k}[1], & \left(a_{k}[i]=P_{k}\left(b_{k}[i]\right)\right) \\
\vdots & \\
b_{n}[0] & \left(b_{n}[i]=b_{n}[i+1]-a_{n-1}[i+1]\right)
\end{array}
$$

By applying cyclic shifts to the Permutations we can get to $b_{k}[0]=0$ to conform with the above definition. Unfortunately the period of the output will be about $M^{n-1}$. Hence, this attack will often not be realizable.

There is still another possibility for a chosen plaintext attack: All previously discussed attacks tried to guess the last rotor. Now we try to attack the first rotor at the beginning. We found that this attack has almost no chance to break the machine and so this will only be a short sketch of how the attack works. With a short random sequence we put the machine into an undefined state. The we keep $b_{1}[i]=x$ constant and look for the length of an output cycle. After doing this several time we can try to classify $P_{1}(x)$ and get $\operatorname{gcd}\left(M, P_{1}(x)\right)$. Having classified all numbers we can try to classify sums of two or more numbers. Continuing with these classifications we can compute the first rotor. The main problem of this attack is that we found no way to make any classifications if more than 4 rotors are used. Another problem is that the length of the output cycles is very big (about $M^{n-1}$ ). So in most cases this attack will be useless. 


\subsection{Chosen-ciphertext-attack}

The basic idea for this attack is to turn the last rotor step by step. It is easy to see that it is sufficient to find a short plaintext sequence that does nothing but turn the last rotor. We will use the 'constant ciphertext effect' mentioned before to find such a sequence. It is sufficient to turn the last rotor for an amount of steps that is coprime to $M$, because every primitive element can be chosen as the ' 1 ' element.

Method: We first decipher the ciphertext $0^{n} 1^{n}$ and get a plaintext $d[1], \ldots, d[2 n]$. The only difference in the state of the machine after deciphering $0^{n}$ and $0^{n} 1^{n}$ is the position of the last rotor. Now the newly constructed plaintext is: $d[1], \ldots, d[n],(d[n+1], \ldots, d[2 n])^{M-1}$ After each $n$-character block the machine is in the same state with the exception that the last rotor has turned for a constant number of steps. The number of steps is $s:=P_{n}^{-1}(0)-P_{n}^{-1}(1)$. The output will be a sequence of the form: $\left(P_{n}(x)\right)^{n}\left(P_{n}(x+s)\right)^{n}\left(P_{n}(x+2 s)\right)^{n} \ldots\left(P_{n}(x+(M-1) s)\right)^{n}$, where $x:=P_{n}^{-1}(0)$ If $s$ is a unit in $\left(Z_{M},+\right)$ the output will cover whole $Z_{M}$ and give the last permutation (except for some isomorphisms as mentioned above). If $s$ is not a unit in $\left(Z_{M},+\right)$ the output will cover only a part of $Z_{M}$. In this case we try an other ciphertext e.g. $0^{n} 2^{n}$. After the last rotor is known we can attack the next rotor. Finally we compute the initial position of the rotors as shown above. This attack is very efficient and depends only polynomially on the values of $n$ and $M$.

\section{Conclusion}

The implementation found in some operating systems uses $n=3$ and $M=256$; i.e. byte encryption with 3 rotors. It can be easily broken with a known plaintext using equations ( 256 unknowns). It seems that, if 5 оге more rotors are used, the only way to break the cipher is a choosen ciphertext attack. So this cipher is, in many environments, a tool to provide good (not optimal) security. A further advantage of this cipher is that it can be implemented very efficiently in both hardware and software. 